\title{
Does one pill a day keep the colonoscopist away?
}

\author{
Alaa Rostom $\mathrm{MD}^{1}$, Paul C Adams $\mathrm{MD}^{2}$, Editor-in-Chief
}

\begin{abstract}
A laa Rostom is a gastroenterologist, clinical epidemiologist and Associate Professor of medicine and community health sciences at the University of Calgary (Calgary, Alberta). He has an interest in the effects of acetylsalicylic acid (ASA), nonsteroidal antiinflammatory drugs (NSAIDs) and cyclooxygenase-2 (COX-2) inhibitors on the gastrointestinal (GI) tract, and has conducted several meta-analyses on the prevention of NSAID-related GI toxicity. He also recently led an extensive systematic review for the US Preventive Services Task Force on the use of ASA, NSAIDs and COX-2 inhibitors for the chemoprevention of colon cancer $(1,2)$.

PA: Can you define chemoprevention of colorectal carcinoma (CRC)?

AR: Chemoprevention refers to the use of an intervention (drug, dietary supplement, etc) on a regular basis by an individual to prevent or reduce the risk of the development of colorectal cancer. Primary chemoprevention refers to the use of such an intervention in subjects without a history of colon cancer. Secondary chemoprevention refers to the use of the intervention in subjects with a history of a resected colorectal cancer. The population in which the intervention is used is also commonly defined in terms of risk. Average-risk individuals are those who have no risk factors for CRC other than age (older than 50 years). Higher risk indi-

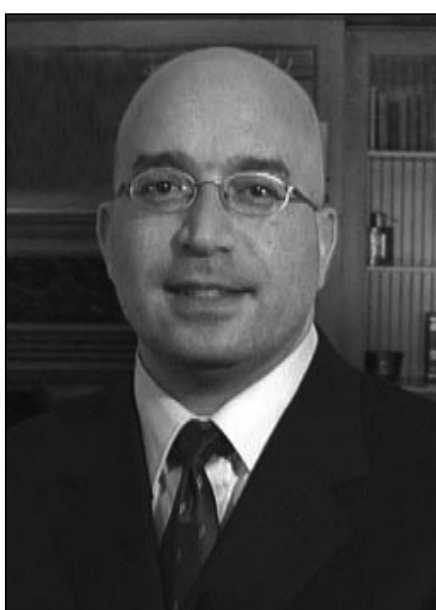

Alaa Rostom is a gastroenterologist, clinical epidemiologist and Associate Professor of medicine and community health sciences at the University of Calgary (Calgary, Alberta). He has an interest in the effect of acetylsalicylic acid, nonsteroidal anti-inflammatory drugs and cyclooxygenase-2 inhibitors on the gastrointestinal tract
\end{abstract}

colorectal adenomas, which are the precursors of most colorectal cancers $(1,2)$.

- This benefit occurs in a dose- and duration-dependent manner. For example, taking ASA every other day in doses similar to those used for the prevention of cardiovascular disease did not reduce the risk of colorectal cancer. The benefits of daily ASA use are most obvious when used at a dose of at least $325 \mathrm{mg}$ and for at least 10 years. Similarly, traditional NSAIDs reduced the risk of colorectal cancer when six to 14 tablets per week were used for more than nine years $(1,2)$.

- Daily and long-term use of ASA and other NSAIDs are associated with important complications:

- ASA, traditional NSAIDs and COX-2 inhibitors all increase the risk of $\mathrm{GI}$ complications such as ulcer bleeding. GI complications with traditional NSAIDs and higher doses of ASA occur in $1.5 \%$ of patients per year. COX-2 inhibitors significantly lower this risk of GI complications compared with traditional NSAIDs by approximately 50\%, but the risks are still approximately five times higher than with placebo $(1,2)$. radic CRC or a personal history of polyps. High-risk individuals are those with a personal history of CRC, or a personal or family history of polyposis or nonpolyposis familial colon cancer syndromes (eg, familial adenomatous polyposis [FAP] and hereditary nonpolyposis colon cancer [HNPCC]).

PA: Is this similar to taking ASA to prevent cardiovascular disease?

AR: Yes, this is analogous to using ASA for the prevention of cardiovascular disease.

PA: When you looked at the risks and benefits of ASA and other NSAIDs for colorectal cancer prevention, what did you find?

AR: The results of our systematic review show that:

- Both ASA and other NSAIDs are effective at reducing the risk of colorectal cancer. The magnitude of the risk reduction is approximately $20 \%$ to $30 \%$. ASA, NSAIDs and COX-2 inhibitors also reduce the risk of

- While ASA can protect against adverse cardiovascular outcomes and death, particularly in the setting of secondary prevention, it can increase the risk of hemorrhagic stroke (1).

- Non-naproxen, traditional NSAIDs and COX-2 inhibitors increase the risk of adverse cardiovascular outcomes, predominantly by increasing the risk of myocardial infarction (2).

PA: How certain can you be that the addition of one new medication for many years is the reason that one group had less colon cancer than the other group?

AR: That is a very important point. Our systematic review included the available randomized, controlled trials, cohort studies and case-control studies. In particular, the observational

\footnotetext{
${ }^{1}$ Division of Gastroenterology, University of Calgary, Calgary, Alberta; ${ }^{2}$ University Hospital, London, Ontario

Correspondence: Dr Alaa Rostom, Division of Gastroenterology, Foothills Hospital, 3330 Hospital Drive Northwest, Calgary, Alberta T2N 4N1. Telephone 403-210-6816, fax 403-210-9368, e-mail arostom@ucalgary.ca

Received and accepted for publication January 21, 2008
} 
designs have various forms of bias that can affect their results. Furthermore, there was variation in how exposure, dose and certain outcomes were ascertained in the observational studies. Nonetheless, we developed an a priori plan to handle this expected variation, and to ensure proper grouping and pooling of studies, when appropriate. The majority of the observational studies reported adjusted rate ratios, taking into account common confounders. We used these adjusted rates rather than the crude rates in the analysis (a list of each study and the adjusted confounders is reported in the appendixes of the two Annals of Internal Medicine papers [1,2]). While the two well-designed randomized, controlled trials of ASA for CRC prevention (physicians and women's health studies) were conducted in relatively healthy subjects, the observational studies addressed a variety of patients at average-risk and higher risk for CRC. So, I am quite confident that the effect of ASA and NSAIDs on adenoma and CRC prevention is real. It is just that the effect is not sufficiently large to justify the added risks.

PA: What did you recommend - are ASA, NSAIDs or COX-2 inhibitors endorsed for CRC chemoprevention?

AR: Based on our findings, we do not recommend the daily long-term use of ASA, traditional NSAIDs or COX-2 inhibitors for the purpose of colorectal cancer prevention in average-risk to 'higher' risk individuals. The harm of these agents appears to outweigh their potential benefit in preventing colorectal cancer, particularly because effective screeningbased strategies are available, which can reduce colorectal cancer incidence and mortality $(1,2)$. The US Preventive Services Task Force also did not recommend the use of these agents for primary CRC chemoprevention (3).

PA: Who do your recommendations apply to?

AR: Our recommendations apply to average-risk individuals (ie, men and women aged 50 years or older), with no personal history of colorectal cancer. Our recommendations also apply to individuals who have a family history of colorectal cancer, and those with a personal history of sporadic colorectal adenomatous polyps. However, our recommendations do not apply to individuals who have already had colorectal cancer themselves, or individuals with a personal or family history of the FAP syndrome or the HNPCC syndromes.

PA: What should people who are not covered by the recommendations do?

AR: Individuals with a history of colorectal cancer, as well as those who have a personal and/or family history of FAP or HNPCC should be enrolled in an adenoma and cancer surveillance program, as defined by a series of guidelines for the screening and management of these patients. The decision to use ASA, NSAID or COX-2 inhibitor chemoprevention in such patients (typically in addition to a surveillance program) should be made on a case-by-case basis in consultation with the patient's attending physician, with consideration of the patient's risks of GI bleeding and cardiovascular disease, as well as the expected benefits of chemoprevention.

PA: How would your recommendations affect the many people taking $81 \mathrm{mg}$ of ASA daily to reduce their risk of cardiovascular disease?

AR: Primary prevention with low-dose ASA reduces the risk of myocardial infarction. In the setting of secondary prevention, low-dose ASA reduces the risk of all-cause mortality, cardiovascular mortality, myocardial infarction and ischemic stroke (1). Therefore, patients with identified indications for low-dose ASA use should continue to use ASA for those indications. However, our findings suggest that low-dose daily ASA use does not appear to protect against colorectal cancer (1). The use of higher doses of ASA further increases the risk for GI complications and hemorrhagic stroke. Therefore, patients currently taking low-dose ASA should resist the temptation to take higher doses for the purpose of colorectal chemoprevention.

PA: There are many dietary supplements promoted to prevent cancer. Can you comment on calcium and other supplements to prevent colon cancer?

AR: Several dietary supplements have been looked at for CRC prevention. A recent meta-analysis in the Journal of the American Medical Association (4) found that dietary fibre intake was inversely associated with CRC cancer risk, but this effect was lost after adjustments were made for other dietary factors such as folate intake. Similarly, dietary carotenoids were not found to prevent CRC in another recent systematic review (5). Physical activity, on the other hand, appears to offer protection from CRC (6), and dietary calcium appears to show modest protection from colonic adenomas (7).

PA: Does fine wine prevent colon cancer?

AR: Unfortunately, regular alcohol intake appears to increase the risk of CRC (8). However, I suspect that the fine quality wine that the editor of The Canadian Journal of Gastroenterology has access to is an entirely different beast!

PA: Do you know any gastroenterologists who are taking pills to prevent colon cancer?

AR: We would all be too embarrassed to call in one of our colleagues when we get a GI bleed from one of these drugs.

\section{REFERENCES}

1. Dube C, Rostom A, Lewin G, et al; US Preventive Services Task Force. The use of aspirin for primary prevention of colorectal cancer: A systematic review prepared for the US Preventive Services Task Force. Ann Intern Med 2007;146:365-75.

2. Rostom A, Dube C, Lewin G, et al; US Preventive Services Task Force. Nonsteroidal anti-inflammatory drugs and cyclooxygenase-2 inhibitors for primary prevention of colorectal cancer: A systematic review prepared for the US Preventive Services Task Force. Ann Intern Med 2007;146:376-89.

3. US Preventive Services Task Force. Routine aspirin or nonsteroidal anti-inflammatory drugs for the primary prevention of colorectal cancer: US Preventive Services Task Force recommendation statement. Ann Intern Med 2007;146:361-4.

4. Park Y, Hunter DJ, Spiegelman D, et al. Dietary fiber intake and risk of colorectal cancer: A pooled analysis of prospective cohort studies. JAMA 2005;294:2849-57.

5. Mannisto S, Yaun SS, Hunter DJ, et al. Dietary carotenoids and risk of colorectal cancer in a pooled analysis of 11 cohort studies. Am J Epidemiol 2007;165:246-55.

6. Samad AK, Taylor RS, Marshall T, Chapman MA. A meta-analysis of the association of physical activity with reduced risk of colorectal cancer. Colorectal Dis 2005;7:204-13.

7. Weingarten MA, Zalmanovici A, Yaphe J. Dietary calcium supplementation for preventing colorectal cancer and adenomatous polyps. Cochrane Database Syst Rev 2004;CD003548.

8. Driver JA, Gaziano JM, Gelber RP, Lee IM, Buring JE, Kurth T. Development of a risk score for colorectal cancer in men. Am J Med 2007;120:257-63. 


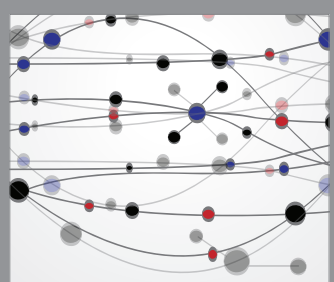

The Scientific World Journal
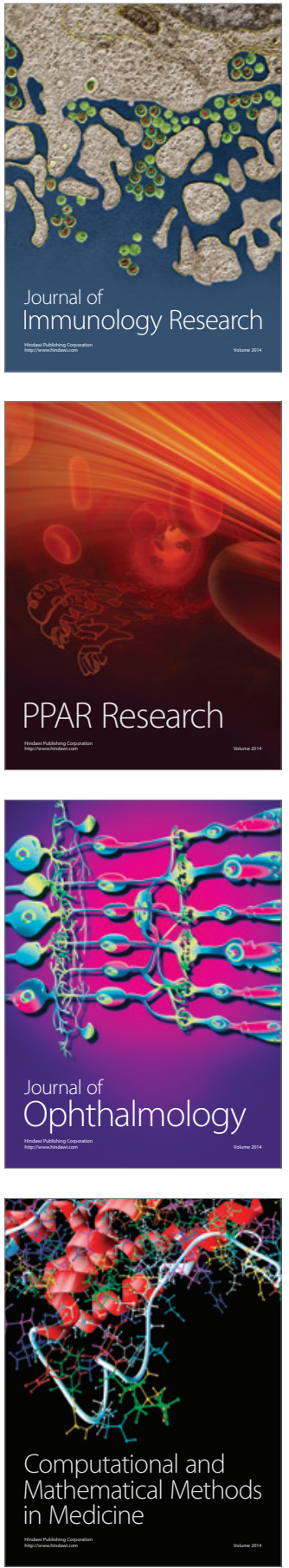

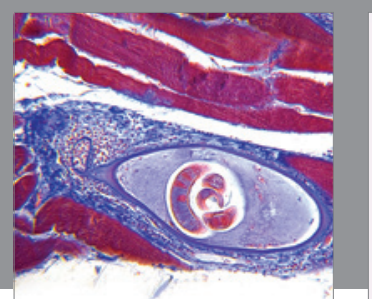

Gastroenterology Research and Practice

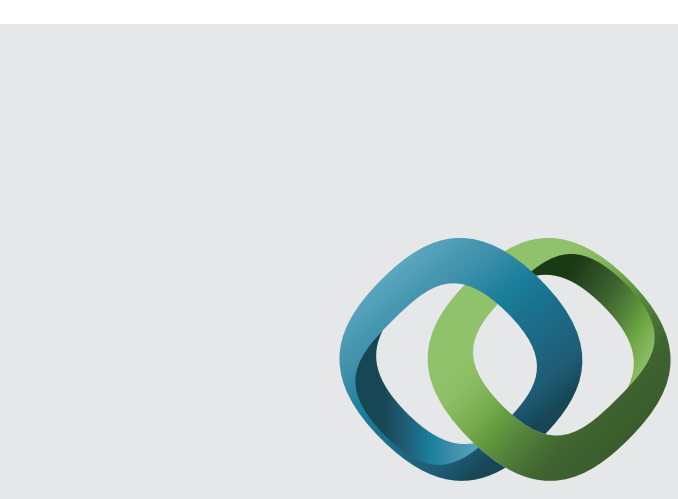

\section{Hindawi}

Submit your manuscripts at

http://www.hindawi.com
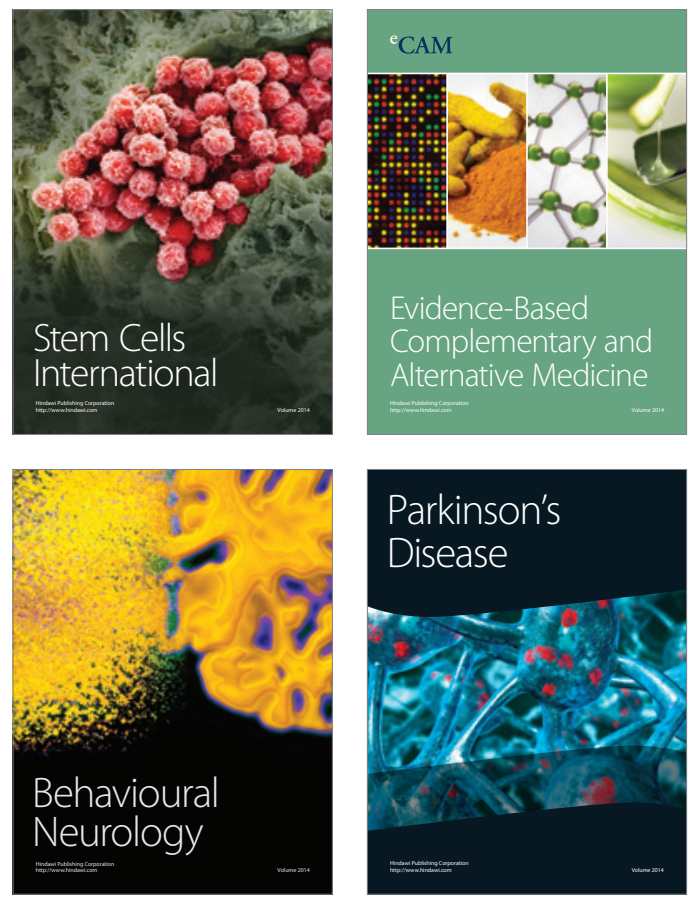
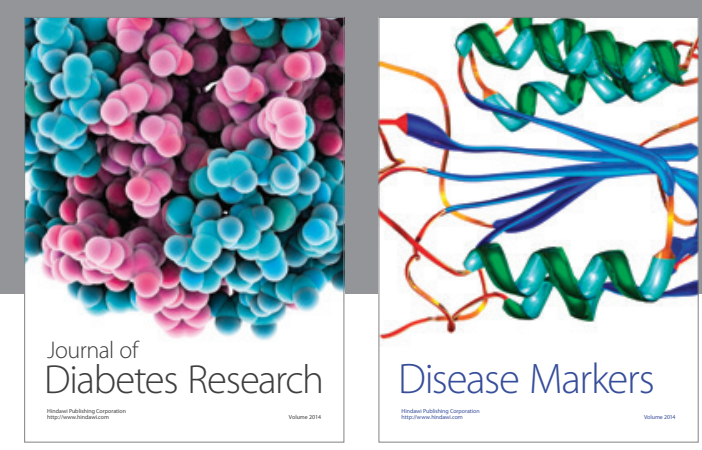

Disease Markers
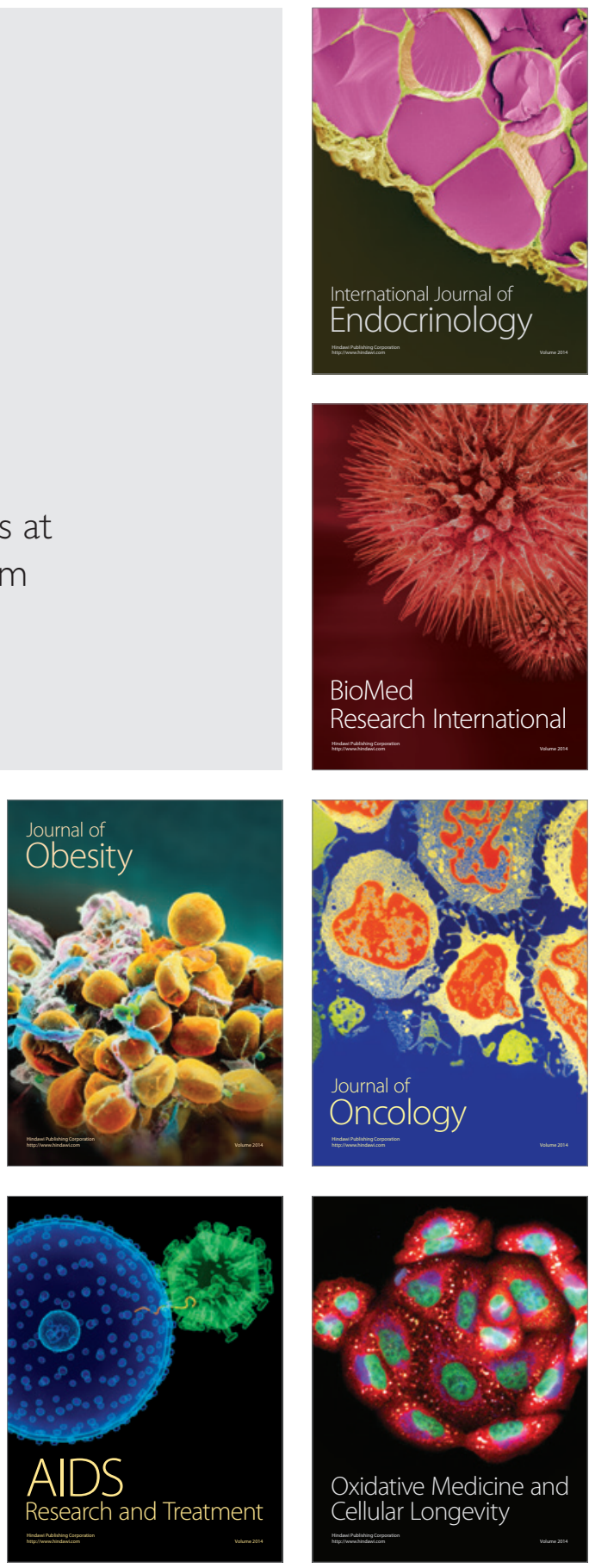